\title{
The Association between Self-Monitored Reproductive Parameters, Pregnancy Outcomes and Acupuncture Treatment
}

\author{
Mayumi Watanabe ${ }^{1 * \#, ~ Y o s h i n o b u ~ N a k a m u r a ~}{ }^{2 \#}$, Chikako Tomiyama ${ }^{3}$, Haiyan Fu ${ }^{1,4}$, \\ Minako Shimaya ${ }^{1}$, Yiwei Ling5, Toru Abo6 \\ ${ }^{1}$ Departmentof Medical Informatics, Niigata University Medical and Dental Hospital, Niigata, Japan \\ ${ }^{2}$ Nakamura Seikotsuin, Niigata, Japan \\ ${ }^{3}$ Department of Medical Technology, Graduate School of Health Sciences, Niigata University, Niigata, Japan \\ ${ }^{4}$ Medical Record Room, Fourth Hospital of Hebei Medical University, Shijiazhuang, China \\ ${ }^{5}$ Department of Bioinformatics, Niigata University Medical and Dental Hospital, Niigata, Japan \\ ${ }^{6}$ Toru Abo Research Centre, Niigata, Japan \\ Email: *watanabem62@gmail.com
}

Received 17 March 2016; accepted 24 May 2016; published 27 May 2016

Copyright (C) 2016 by authors and Scientific Research Publishing Inc.

This work is licensed under the Creative Commons Attribution International License (CC BY). http://creativecommons.org/licenses/by/4.0/

c) (i) Open Access

\begin{abstract}
Many women track their daily basal body temperature (BBT), an important factor in reproductive status, as a part of fertility self-management. This study involved 135 patients who had undergone conventional infertility treatment; they had weekly acupuncture treatment and we assessed BBT, menstrual cycle, menstrual period and menstrual bleeding amount pre- and post-treatment. We also analyzed the rate of insufficient description of reproductive parameters, comparing patients who had delivered a baby with those who had a miscarriage and those who did not become pregnant. Menstrual cycle and period did not show notable changes pre- and post-acupuncture treatment in all groups. Menstrual bleeding amount decreased in the no-pregnancy group. There was a suggestion that acupuncture decreased very long menstrual cycle (e.g. bimonthly) to monthly. The insufficient description rate of menstrual bleeding amount was high in all groups. Weekly discussion about monitoring reproductive parameters could improve patients' awareness in self-management. To prevent miscarriage patients needed psychological and medical advice for careful self-management in pregnancy and menstrual cycle.
\end{abstract}

\section{Keywords}

Basal Body Temperature Chart, Pregnancy, Miscarriage, Menstrual Cycle, Insufficient

\footnotetext{
*Corresponding author.

${ }^{\#}$ MW and YN contributed equally to this work.
} 


\section{Description}

\section{Introduction}

The consequences of infertility can have negative impacts on women's lives because the conflux of personal, interpersonal, social, and religious expectations can bring a sense of failure, loss, and exclusion to those who are infertile [1] [2].

In the 1920s, Dr. Kyusaku Ogino established a technique to identify the date of ovulation to help women time intercourse to achieve pregnancy [3]. This discovery paved the way for modern reproductive treatment. Many women track their basal body temperature (BBT) every day, as it is an important indicator for reproduction and a useful tool for effective self-management.

The current study aimed to use a specialized chart to track the reproductive parameters BTT, menstrual cycle, menstrual period and bleeding amount in women who had undergone conventional fertility treatment. As compliance with completing the charts was not $100 \%$, we also analyzed the differences in missing data between the delivery, miscarriage and no-pregnancy groups.

\section{Materials and Methods}

\subsection{Patients}

We studied the BBT chart of 135 patients who regularly attended Nakamura Seikotsuin (Niigata, Japan) and Yushima Shimizuzaka Clinic (Tokyo, Japan) for two years between March 1997 and December 2015. All patients had undergone conventional infertility treatment for more than 2 years by medical specialists before commencing acupuncture treatment. Written informed consent was obtained from all subjects and the study was approved by the Medical Ethics Committee of Yushima Shimizuzaka Clinic.

\subsection{Acupuncture (Dizhen Treatment)}

An ancient shaped acupuncture needle (made of stainless steel) Dizhen (DZ; Soken Medical Co. Ltd., Toshima, Japan), was used for the acupuncture treatment. As DZ use is considered to be advantageous and safe in modern clinical practice, it can avoid the issues with infection and tissue damage sometimes seen with modern acupuncture needles [4] [5]. The acupuncture application points corresponded to the Conception Vessel, Governor Vessel and Bladder Meridian as defined by the World Health Organization [6]. The treatment was performed by nationally licensed experienced Japanese acupuncturists.

\subsection{Assessment of Basal Body Temperature}

Participants were asked to measure and record BBT under the tongue with a basal thermometer immediately after waking every morning (after at least three hours of uninterrupted sleep).

\subsection{Analysis of Menstrual Cycle, Period, and Bleeding Amount}

Participants recorded the number of days between menses onsets (menstrual cycle), number of days of menstruation (menstrual period) and menstrual bleeding amount, which was subjectively assessed with a novel method of quantification developed by our group. Bleeding amount was scored as either heavy bleeding level (10 points), ordinary bleeding level ( 5 points) or light bleeding level (1 point). The amount of menstrual bleeding during one menstrual period was then calculated as a sum of these scores (Figure 1).

\subsection{Statistical Analysis}

The Mann-Whitney test was conducted and the level of significance was set at $p<0.05$. The values presented were expressed as range as well as mean \pm SD. Where data were unavailable, we calculated the rate of insufficient description. All statistical analyses were performed using SPSS Statistics for Windows version 20.0 (IBM Corp, Armonk, NY). 
(a) basal body temperature chart

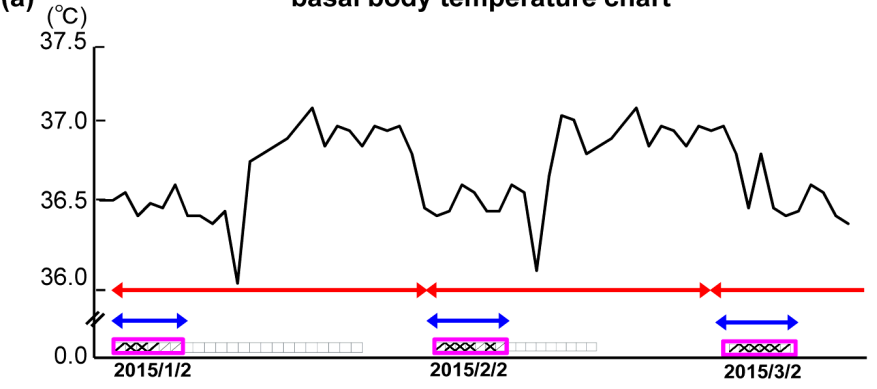

(b)

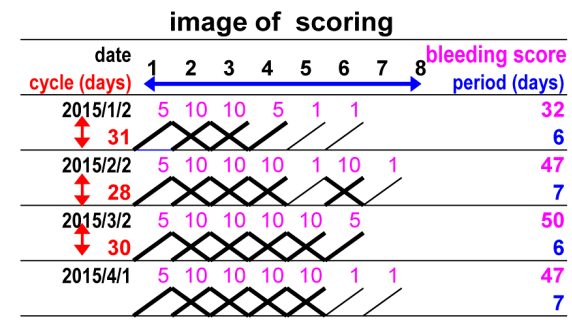

Figure 1. A representative example of a basal body temperature chart including an image of the scoring of menstrual bleeding amount (pink).

\section{Results}

Patients were divided into three groups: a delivery group $(n=57$; age range 21 - 47 years; age mean $32.5 \pm 5.3$ years), a miscarriage group $(n=36 ; 21-45 ; 35.7 \pm 5.5)$ and a no-pregnancy group $(n=42 ; 21-42 ; 31.2 \pm 4.8)$. The first group became pregnant after acupuncture treatment (on average $23 \pm 25$ months later). The second group became pregnant but had a miscarriage. Patients in the third group did not become pregnant despite attending the clinic regularly for 2 years. Details of patients and groups are shown in Figure 2.

\subsection{Menstrual Cycle}

There was no difference in menstrual cycle between pre- and post-acupuncture treatment in the delivery group (from $31.9 \pm 6.9$ to $30.9 \pm 5.3$ days) and no-pregnancy group (from $30.7 \pm 8.2$ to $30.6 \pm 6.7$ days) (Figure 3(a)). Participants in the miscarriage group did not complete BBT charts pre-acupuncture treatment; post-treatment their average menstrual cycle was $31.4 \pm 5.3$ days. Weekly acupuncture decreased the distribution range of menstrual cycle in the no-pregnancy group (from 17 - 77 to 13 - 52 days) while that in the delivery group did not change (from 20 - 49 to 24 - 50 days).

\subsection{Menstrual Period}

Acupuncture had no effect on menstrual period in all groups. The average length of menstrual period was $5.6 \pm$ 1.2 in the delivery group, $5.7 \pm 1.3$ in the miscarriage group and $5.8 \pm 1.5$ in the no-pregnancy group (Figure $3(\mathrm{~b}))$.

\subsection{Menstrual Bleeding Amount}

The no-pregnancy group showed a significant decrease in menstrual bleeding amount post-acupuncture treatment compared with pre-treatment (from $45.2 \pm 13.1$ to $37.4 \pm 7.9, p=0.013$ ). Such differences were not found in other groups (Figure 3(c)).

\subsection{Insufficient Description Rate}

Interestingly, participants in the miscarriage group did not record BBT pre-acupuncture, with an insufficient description rate of $100 \%$. After 2 years this rate decreased to $33.3 \%$. In other words, $66.7 \%$ of participants in this group still did not record BBT (Figure 4). 
patients who experienced conventional infertility treatment

more than two years by medical specialists $(n=135)$

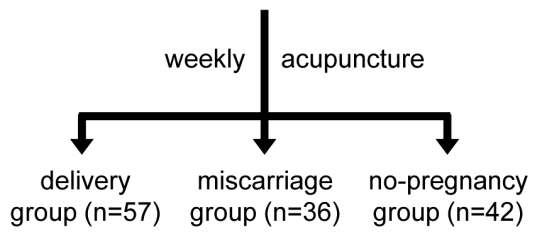

Figure 2. Overview of the 135 patients and three groups: delivery, miscarriage and no-pregnancy.

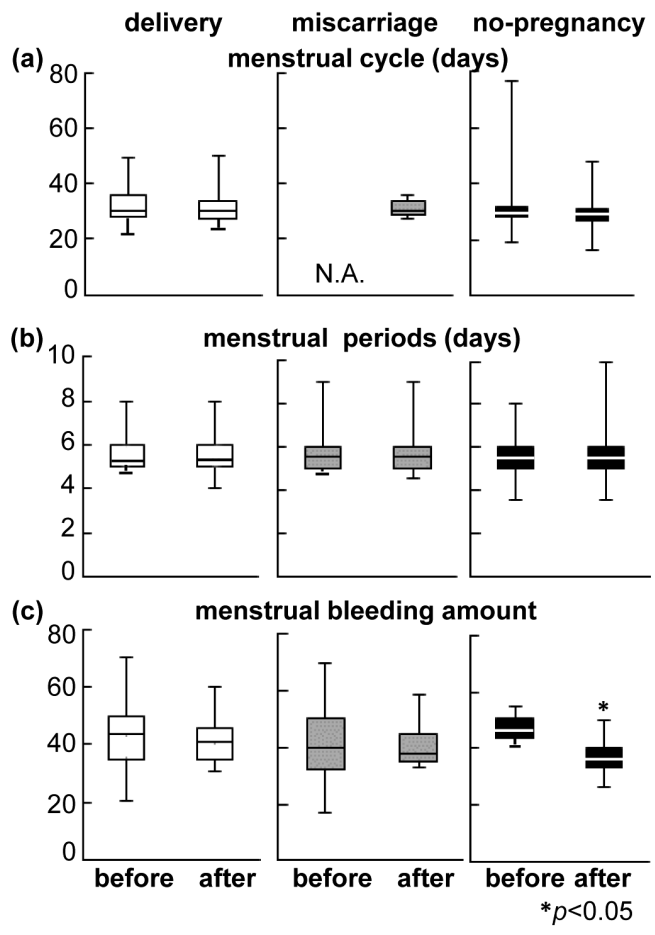

Figure 3. (a) Menstrual cycle, (b) menstrual period and (c) menstrual bleeding amount in the three groups. Compared with pre-acupuncture treatment, menstrual bleeding amount score decreased in the no-pregnancy group post-treatment $\left({ }^{*} p<0.05\right)$.

The rate of insufficient description of menstrual cycle was also higher in the miscarriage group both pretreatment (miscarriage vs. delivery and no-pregnancy groups: $38.9 \%$ vs. $4.8 \%$ and $3.5 \%$ ) and post-treatment (27.8\% vs. $2.4 \%$ and $12.3 \%$ ).

All groups showed a high rate of insufficient description in menstrual bleeding amount; this did not change between pre- and post-acupuncture treatment.

\section{Discussion}

\subsection{Regular Discussion of BBT Could Improve Self-Management}

There have been reports that acupuncture is an effective treatment for infertility; the regulation of the hypothalamic-pituitary-ovarian axis has been implicated in this effect [7]-[11].

In the current study, all patients had undergone conventional infertility treatment for more than 2 years before commencing acupuncture treatment. However, acupuncture treatment was not associated with a statistically significant difference in menstrual cycle or menstrual period, possibly due to high inter-subject variability. Nevertheless, 77 patients out of 135 who had given up conventional fertility treatment were able to deliver safely. 


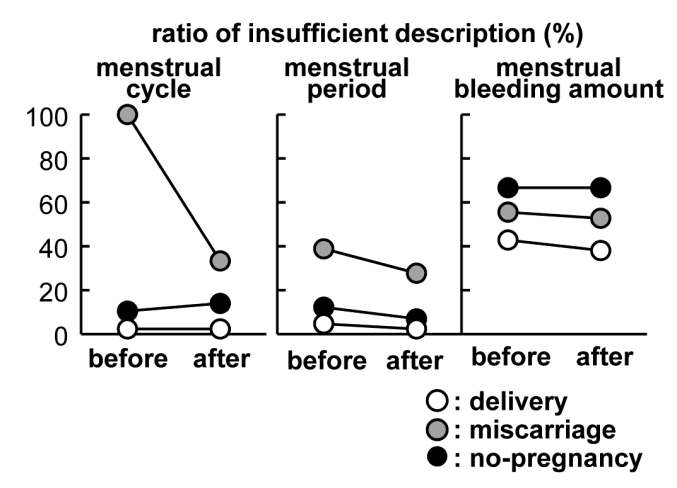

Figure 4. Rates of insufficient description of menstrual cycle, menstrual period and menstrual bleeding amount. Rates of insufficient description of menstrual cycle were $100 \%$ (pre-acupuncture) and $66.7 \%$ (post-acupuncture) in the miscarriage group.

It may be possible to raise self-management awareness through weekly discussion of BBT as well as acupuncture treatment.

\subsection{Psychological and Medical Advice Is Needed in Pregnancy Self-Management}

The miscarriage group showed poorer self-reporting of reproductive parameters than the other groups. They did not provide information on menstrual cycle while only $61.1 \%$ tracked menstrual period. One problem may be found in their sustainability in BBT tracking, especially in more than 2 months.

Menstrual cycle was defined as the length of time between the onsets of menses for 2 consecutive months. Accordingly, while menstrual period can be determined in only one month, determining menstrual cycle requires 2 months. This indicates that participants in the miscarriage group did not self-monitor for more than 2 months before commencing acupuncture treatment. Even after completing 2 years of treatment, $66.7 \%$ did not consistently track BBT. This study suggests that self-management of those in the miscarriage group was poorer than that of other groups.

Shimaya et al. reported that a unique Japanese concept "amae" (a kind of dependency) needs to be considered in the case of outpatients who poorly manage themselves (e.g. in taking immunosuppressive medicines after kidney transplantation) [12]. Participants in the miscarriage group were able to become pregnant, but were not able to continue to term. Minagawa et al. reported that during pregnancy women are in a state of sympathetic nerve dominance, suggesting that pregnant women need careful self-management until delivery [13]. For example, one of our patients, who was observed in this study, had a miscarriage soon after exercising in a cold water pool, possibly due to a cold stress-induced imbalance in autonomic nerve status (excess sympathetic nerve dominance) via hypothermia. Accordingly, our patients were advised to avoid stressful situations (e.g., overwork) and the use of hot-water bottles while sleeping, and advised to practice deep breathing several times a day to promote relax [14]. Psychological advice from a specialist, as well as medical support, may be beneficial for pregnant women.

\subsection{Menstrual Bleeding Amount Decreased in the No-Pregnancy Group Post-Treatment}

The method for scoring menstrual bleeding amount is original to this study. Despite inter-subject variability, menstrual bleeding amount decreases in the no-pregnancy group between pre- and post-acupuncture treatment. One explanation for this can be that, as average age in this group (31.2 \pm 4.8 ) is younger than other groups (32.5 \pm 5.3 in the delivery group and $35.7 \pm 5.5$ in the miscarriage group), acupuncture treatment may be more effective in younger women. Alternatively, some patients have a very long menstrual cycle (up to 77 days) pre-acupuncture treatment; this duration decreases to 52 days indicating that menstrual cycles of some patients change from bimonthly to monthly. Women have two ovaries (right and left) which release an egg in alternate months, therefore we suggest that a dysfunctional ovary may have recovered its function of regulating the amount of bleeding. However, further study is needed to support this hypothesis. 
Our study had several limitations. Firstly, we studied only patients who had undergone conventional infertility treatment for more than 2 years. Therefore, we studied neither healthy patients nor patients who did not present BBT. Secondly, while female factors accounted for $51 \%$ of infertility cases, $19 \%, 18 \%$ and $12 \%$ were due to male factors, a combination of male and female factors, and unexplained causes respectively [15]. Moreover, there were gender differences in physical and mental awareness in young people [16] [17]. Future research into reproductive problems in males could focus on psychological aspects. Thirdly, as the current study was relatively small, larger comparative studies exploring the social and cultural backgrounds of other countries, the correlation between age and test index was needed to add support to its conclusions.

\section{Acknowledgements}

Special thanks go to Mr Taiki Hashimoto and Ms Kaori Yamamoto (Yushima-Shimizuzaka Clinic) for arrangement of the clinical research. The authors also thank all of the patients who participated in this study.

\section{References}

[1] Fatima, P., Rahman, D., Hossain, H.B., Hossain, H.N. and Mughi, C.R. (2015) Psychosocial Consequences of Infertility on Infertile Women. Mymensingh Medical Journal, 24, 704-709.

[2] Rutstein, S.O. and Shah, I.H. (2004) Infecundity, Infertility, and Childlessness in Developing Countries. DHS Comparative Reports, 9. http://www.nhs.uk/conditions/contraception-guide/pages/contraceptive-implant.aspx

[3] Kippley, J.F. (2008) Sexual Revolution Part II. Catholic Social Science Review, 13, 373-386. http://dx.doi.org/10.5840/cssr20081332

[4] Watanabe, M., Takano, O., Tomiyama, C., Matsumoto, H., Urahigashi, N., Kainuma, E., Madarame, T., Fukuda, M. and Abo, T. (2012) The Effects of Application of an Ancient Type of Acupuncture Needle on Body Temperature, Immune Function and the Autonomic Nerve System. Health, 4, 775-780. http://dx.doi.org/10.4236/health.2012.410120

[5] Watanabe, M., Takano, O., Tomiyama, C., Guan, J., Hou, G., Mori, H., Nishijo, K., Abo, T. and Akazawa, K. (2013) The Effects of Application of an Ancient Type of Acupuncture Needle on Increase in Urination of Hospitalized Oldest-Old People. Health, 5, 1092-1098. http://dx.doi.org/10.4236/health.2013.57147

[6] World Health Organization (2002) Acupuncture: Review and Analysis of Reports on Controlled Clinical Trials. World Health Organization, Geneva.

[7] World Health Organization (2003) Acupuncture: Review and Analysis of Reports on Controlled Clinical Trials. World Health Organization, 87.

[8] Manheimer, E., Zhang, G., Udoff, L., Haramati, A., Langenberg, P., Berman, B.M. and Bouter, L.M. (2008) Effects of Acupuncture on Rates of Pregnancy and Live Birth among Women Undergoing in Vitro Fertilisation: Systematic Review and Meta-Analysis. BMJ, 336, 545-549.

[9] Boivin, J., Griffiths, E. and Venetis, C.A. (2011) Emotional Distress in Infertile Women and Failure of Assisted Reproductive Technologies: Meta-Analysis of Prospective Psychosocial Studies. BMJ, 342. http://dx.doi.org/10.1136/bmj.d223

[10] Louis, G.M., Lum, K.J., Sundaram, R., Chen, Z., Kim, S., Lynch, C.D., Schisterman, E.F. and Pyper, C. (2011) Stress Reduces Conception Probabilities across the Fertile Window: Evidence in Support of Relaxation. Fertility and Sterility, 95, 2184-2189. http://dx.doi.org/10.1016/j.fertnstert.2010.06.078

[11] Akbarzadeh, M., Masoudi, Z., Zare, N. and Vaziri, F. (2015) Comparison of the Effects of Doula Supportive Care and Acupressure at the BL32 Point on the Mother's Anxiety Level and Delivery Outcome. Iranian Journal of Nursing and Midwifery Research, 20, 239-246.

[12] Shimaya, M., Watanabe, M., Azumi, M., Shichiri, K., Tomiyama, C., Tanabe, M., Sato, S. and Akazawa, K. (2015) A Questionnaire Survey in Kidney Transplant Outpatients: Factors Associated with Good Self-Management. Health, 7, 589-595. http://dx.doi.org/10.4236/health.2015.75070

[13] Minagawa, M., Narita, J., Tada, T., Maruyama, S., Shimizu, T., Bannai, M., Oya, H., Hatakeyama, K. and Abo, T. (1999) Mechanisms Underlying Immunologic States during Pregnancy: Possible Association of the Sympathetic Nervous System. Cellular Immunology, 196, 1-13. http://dx.doi.org/10.1006/cimm.1999.1541

[14] Watanabe, M., Miyajima, K., Matsui, I., Tomiyama-Miyaji, C., Kainuma, E., Inoue, M., Matsumoto, H., Kuwano, Y. and Abo, T. (2010) Internal Environment in Cancer Patients and Proposal That Carcinogenesis Is Adaptive Response of Glycolysis to Overcome Adverse Internal Conditions. Health, 2, 781-788. http://dx.doi.org/10.4236/health.2010.27118

[15] Ontario Ministry of Children and Youth Services (2013) Care to Proceed: Infertility and Assisted Reproduction in On- 
tario. http://www.children.gov.on.ca/htdocs/English/infertility/report/caretoproceed.aspx

[16] Shichiri, K., Shibuya, M., Murayama, K., Sato, C., Kaminushi, K., Uenoyama, T., Mashima, I., Kuroda, T. and Suzuki, Y. (2015) Features of Developmental Level of Defense Mechanisms and Adjustment Status of University Students in Japan. Health, 7, 52-57. http://dx.doi.org/10.4236/health.2015.71007

[17] Shichiri, K., Shibuya, M., Watanabe, M., Tahashi, M., Kaminushi, K., Uenoyama, T., Mashima, I., Murayama, K., Kuroda, T. and Suzuki, Y. (2016) Correlations between the Profile of Mood States (POMS) and the WHOQOL-26 among Japer.. 